\title{
Classification
}

Physics Abstracts

$05.50-64.60 \mathrm{H}-75.40 \mathrm{D}$

\section{Energy barriers of the Ising model on percolation clusters : a numerical study}

\author{
R. Rammal and A. Benoit \\ Centre de Recherches sur les Très Basses Températures, CNRS, B.P. 166 X, \\ 38042 Grenoble Cedex, France
}

(Reçu le 15 mai 1985, accepté sous forme définitive le 11 juin 1985)

\begin{abstract}
Résumé. - On décrit un nouvel algorithme efficace pour déterminer la barrière d'énergie de retournement d'un amas fini de spins d'Ising ferromagnétiques. Cette méthode est illustrée dans le cas des amas de percolation au seuil. On montre que la barrière augmente comme le logarithme de la taille au lieu d'une loi de puissance bien connue dans les réseaux euclidiens. Certaines conséquences de ces résultats pour le modèle d'Ising cinétique sur les ferromagnétiques dilués sont brièvement discutées.

Abstract. - A new efficient algorithm for determining the energy barrier to overturn a finite cluster of ferromagnetic Ising spin is described. Percolation clusters at threshold are used to illustrate our method. We show in particular that the energy barrier scales logarithmically with the cluster size in contrast with the known power law behaviour on Euclidean lattices. Some of the consequences of this result for the kinetic Ising model on diluted ferromagnets are briefly discussed.
\end{abstract}

\section{Introduction.}

In this Letter we report on some new results pertaining to the dynamics of diluted ferromagnets. As a prototype we consider the ferromagnetic Ising model on a diluted lattice. Although the static critical behaviour is now well established [1], the dynamics of this model is still quite unknown. Here we attempt to correct this situation. Among various questions the following one is of prior importance : how is the spin dynamics modified when the percolation threshold $p_{\mathrm{c}}$ is approached ? Such a question is motivated by the fact that at threshold, both the infinite cluster and its backbone are self-similar (i.e. fractals). In the past few years, the influence of scale invariance (dilation symmetry) on the physics of fractals has been the subject of intensive studies. For instance, the anomalous behaviour of physical properties (classical diffusion, spectrum of the discrete Laplacian operator, elementary excitations, localization, ...) has been pointed out by different authors [2,3]. However, all these problems are linear and the role of the spectral dimension appears to be dominant [4]. In contrast, the kinetic Ising model [5] is inherently a non-linear problem. Therefore, the dynamics of Ising spin models on self-similar lattices provides a new physical problem, potentially relevant to physical applications such as neutron scattering experiments [6] on diluted magnets.

As usual, the key role of our analysis is played by the physical behaviour on finite percolation clusters at threshold $\left(p=p_{\mathrm{c}}\right)$. Relevant results at $p<p_{\mathrm{c}}$ and $p>p_{\mathrm{c}}$ are simply deduced by using scaling arguments and averaging over the cluster size distribution [7]. Therefore we will 
focus our attention here to finite clusters at threshold. More detailed results will be published elsewhere [8].

\section{Low temperature approach.}

In the following, we consider the Ising model, defined by the Hamiltonian $\mathscr{H}=-\sum_{\langle i j\rangle} J_{i j} \sigma_{i} \sigma_{j}$ on a diluted lattice. Here $\sigma_{i}= \pm 1$ and $J_{i j}=J$ (if both the nearestneighbour sites $i$ and $j$ are occupied) or $J_{i j}=0$ (otherwise). This model is known to have no long range order at $p<p_{\mathrm{c}}$ and orders at $T_{\mathrm{c}}(p)$ for $p>p_{\mathrm{c}}$, with $T_{\mathrm{c}}(p) \rightarrow 0$ as $p \rightarrow p_{\mathrm{c}}$. At threshold, the spin-spin correlation length scale is $\xi_{T} \sim \exp \left(2 K \cdot v_{\mathrm{p}}\right)$, where $K=J / T\left(k_{\mathrm{B}}=1\right)$ and $v_{\mathrm{p}}$ is the critical exponent of the percolation transition.

At threshold both infinite and finite clusters are present. Let us consider the Hamiltonian $\mathscr{H}=-\sum_{\langle i j\rangle} J \sigma_{i} \sigma_{j}$ describing $s$ Ising spins on a given cluster. Such a cluster is actually a selfsimilar object, of fractal dimension $d_{\mathrm{p}}=d-\beta_{\mathrm{p}} / \nu_{\mathrm{p}}\left(d=\right.$ space dimension; $\beta_{\mathrm{p}}$ and $v_{\mathrm{p}}$ are the percolation transition exponents). Since this interacting spin system has no intrinsic dynamics, a stochastic approach is usually used [5] in order to mimic the interaction with the heat bath. Within this framework of Glauber dynamics, the time-dependent probability for a given spin configuration satisfies a master equation. The single-spin flip dynamics, satisfying the detailed balance condition, can be described for instance by the transition probability $\omega_{i}\left(\sigma_{i}\right)=\tau_{0}^{-1} \times$ $\left(1-\sigma_{i}\right.$ th $\left.\left(\frac{h_{i}}{T}\right)\right)$ where $h_{i}=-\sum_{j} J_{i j} \sigma_{j}$ is the local field at site $i$.

Except for the 1D case (open or closed chain of spins), the Glauber dynamics leads in general to a hierarchy of non-linear coupled equations. The use of truncation schemes or renormalization group ideas fails to produce reliable results [9]. On the other hand, the direct approach for solving the system of $2^{s}$ linear equations giving the time-dependent probabilities of spin configuration cannot be implemented in practice, particularly for the percolation clusters.

All these difficulties can be surmounted at low temperatures, when $\xi_{T} \gg l$. Here, $l$ is the linear size of the considered cluster $: s \sim l^{d_{\mathrm{p}}}$. Indeed, in this limit, the system spends its time in its two ground states : $\sigma_{i}=+1$ (all $i$ ) or $\sigma_{i}=-1$ (all $i$ ) and the thermal relaxation rate will be dominated by the energy barrier for single spin-flip dynamics. The determination of the energy barrier for a given cluster is therefore the first step in the study of its dynamics.

Before considering some examples, some words of caution are in order. In general, for a system in contact with a heat bath at temperature $T$, the escape from up component to down component is dominated by free energy barriers surrounding each component in phase space and preventing escape. Furthermore, a few low-energy states will permit appreciable escape because of the entropy cost (" entropic barriers "). Therefore, in all cases, we need for small $\exp (-\Delta F / T)$ where $\Delta F$ is the free energy barrier height. In the expression of $\Delta F=\Delta E-T \Delta S$ both the energy cost $\Delta E$ and the entropy cost $\Delta S$ are of importance. Only at very low temperatures, $\Delta E$ can dominate and the relaxation rate will be controlled by the energy barrier alone.

Let us consider a simple example to illustrate this point. For a $d$-dimensional Ising model (Euclidean lattice), the least costly escape path from the up component to the down component involves sweeping a domain wall across the sample of $s$ spins. Both $\Delta E$ and $-\Delta S$ are proportional to the defect volume : $s^{(d-1) / d}$. For $\Delta S$ this comes from the fact that $\sim s^{(d-1) / d}$ degrees of freedom are constrained by the defect. Defect nucleation is a common route by which energy barriers are surmounted. The same argument holds also for the infinite range model $\left(J_{i j} \sim 1 / s\right)$ and $\Delta F \sim s^{2} / s \sim s$. Note that at $d=1, \Delta F \sim 1$ does not depend on the size $s$ of the chain. Therefore, beside this case, $\Delta F$ increases with the system size as a power law. A natural question then arises : what is the corresponding behaviour on percolation clusters and more generally on a self- 
similar structure ? In order to answer this question, we need a very precise definition of the energy barrier for a given system.

\section{Energy barriers : definition and numerical method.}

The energy barrier which controls the thermal relaxation rate for a single spin-flip dynamics can be defined in terms of paths in the phase space. For percolation clusters, the barrier turns out to be very sensitive to the structure details in contrast with the Euclidean case. For a given cluster of $s$ spins, the energy barrier for its reversal from state up to state down will be defined as follows. We reverse the $s$ spins in a given order and find the energy barrier (the maximum energy) for that sequence. There are $s$ ! sequences (paths) between the initial and final states. The cluster barrier $V$ is defined as the smallest energy barrier among the $s !$ paths. Note that such an optimal path is in general not unique.

We have used a fast algorithm to generate permutations by $s$ ! successive interchanges of adjacent elements. This algorithm appears to be optimal for our purpose, up to $s=10$ and has been used [8] for an exact enumeration calculation (small $p$ expansion) for the energy barriers of small clusters. For larger clusters, up to $s \sim 10^{3}$ at $2 \mathrm{D}$ and 200 at 3D, we have used a new efficient algorithm : the Landscape exploration method described below. Note that another method would be a sampling procedure over the $s$ ! possible paths. This can be done for instance by generating a sequence of random permutations instead of the exhaustive list of the total number of paths. Because of the large number of paths, such an approximate procedure is not very accurate and leads to an overestimation of the barrier.

Let us describe the Landscape exploration method in some detail. This is a direct and very general method giving the exact value of the energy barrier. The basic principle is very simple and can be summarized as follows. Starting from the origin $E=0$ of energies, an increasing energy level $E / 2 J=1,2, \ldots$ is defined at each step of the exploration of paths. At a given value of $E$, all the possible sequences of spin reversals, leading to spin configurations of energy lower than $E$, are explored. This exploration step is repeated, with an increasing value of $E$, up to that allowing the reversal of the whole set of spins.

Viewed as a direct method, the Landscape exploration can be adapted to study other problems. In the present case, this procedure can be accelerated according to the following remarks :

a) During an exploration step, each time that a spin flip is feasible at constant energy, then the corresponding reversal is immediately performed. This is for instance the case on a degenerate plateau coming from one-dimensional chains inside the cluster.

b) The energy increment $\Delta \varepsilon$, associated with the flip of a single spin, is lower than $2 J d$ in dimension $d$. This remark permits to find the set of potentially spin reversals at a given energy level. In particular, the spins located at the sites of lowest valence are allowed to flip first.

c) At a given energy level, $E \geqslant 2 J d$, adjacent spins having an energy increment $\Delta \varepsilon \leqslant 2 J d$ are reversed simultaneously.

d) At the beginning of an exploration step, associated with a new value of $E$, if a set of nonadjacent spins of valence lower than $d$ are allowed to flip in a given order, then the same order is maintained for further explorations. Note that an already flipped spin is never allowed to turn back later. In addition, each site of the cluster is checked as a possible origin in the search for an optimal path.

A similar procedure has been used for the calculation of the domain wall energy barrier $W$. In this case, spins flip only when at least one neighbour has flipped, so the flipped domain is constrained to be simply connected at all times. In figure 1 two optimal paths are shown for $V$ and $W$ respectively, for a small cluster $(s=48)$. The corresponding energy profiles are shown in figure 2 . This example illustrates the general property $W \geqslant V$. In fact, as was mentioned before, according 

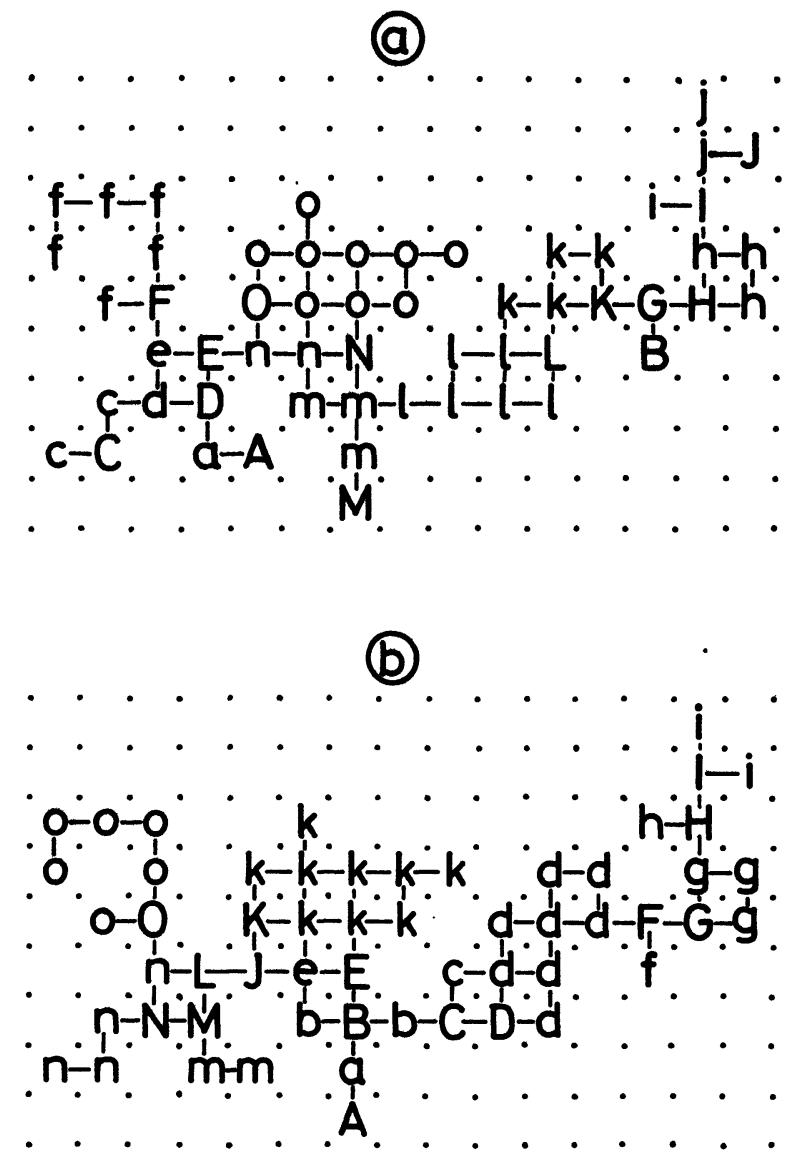

Fig. 1. - The pattern of successive spin flips, giving rise to an optimal path for overturning a cluster of $s=48$ Ising spins on the square lattice. Spin A, flipped first, is followed by spins a, B, C, ... according to alphabetical order. Here (a) refers to the energy barrier path and (b) to that of the domain wall energy barrier.

to a common belief, in order to reverse a block of spins, one must pass a domain wall across the block, with reversed spins behind the wall. The maximum energy of the domain wall is actually the energy barrier $W$. However, it is not difficult to exhibit simple examples where $W>V$. Of course, the equality is reached on Euclidean block. However the optimal path associated with $V$ may be a very subtle one, as seen in figure 1 , and may correspond to disconnected flipped parts of the cluster.

It should be noticed, however, that it is much easier to calculate $W$ than $V$. Furthermore, the difference $W-V$ may be very small and this is actually the general case. For instance, for a particularly large cluster $(s=1417)$, we have obtained $V / 2 J=8$ and $W / 2 J=9$ respectively. Therefore, the optimal path for overturning the cluster is not necessarily associated with a domain wall. More subtle paths can exist and the corresponding barrier is actually the relevant one for the dynamics.

\section{Numerical results.}

Using the Landscape exploration method, we have performed a Monte Carlo calculation of the energy barrier distribution for both 2D and 3D percolation clusters. Clusters of size $s$ were 

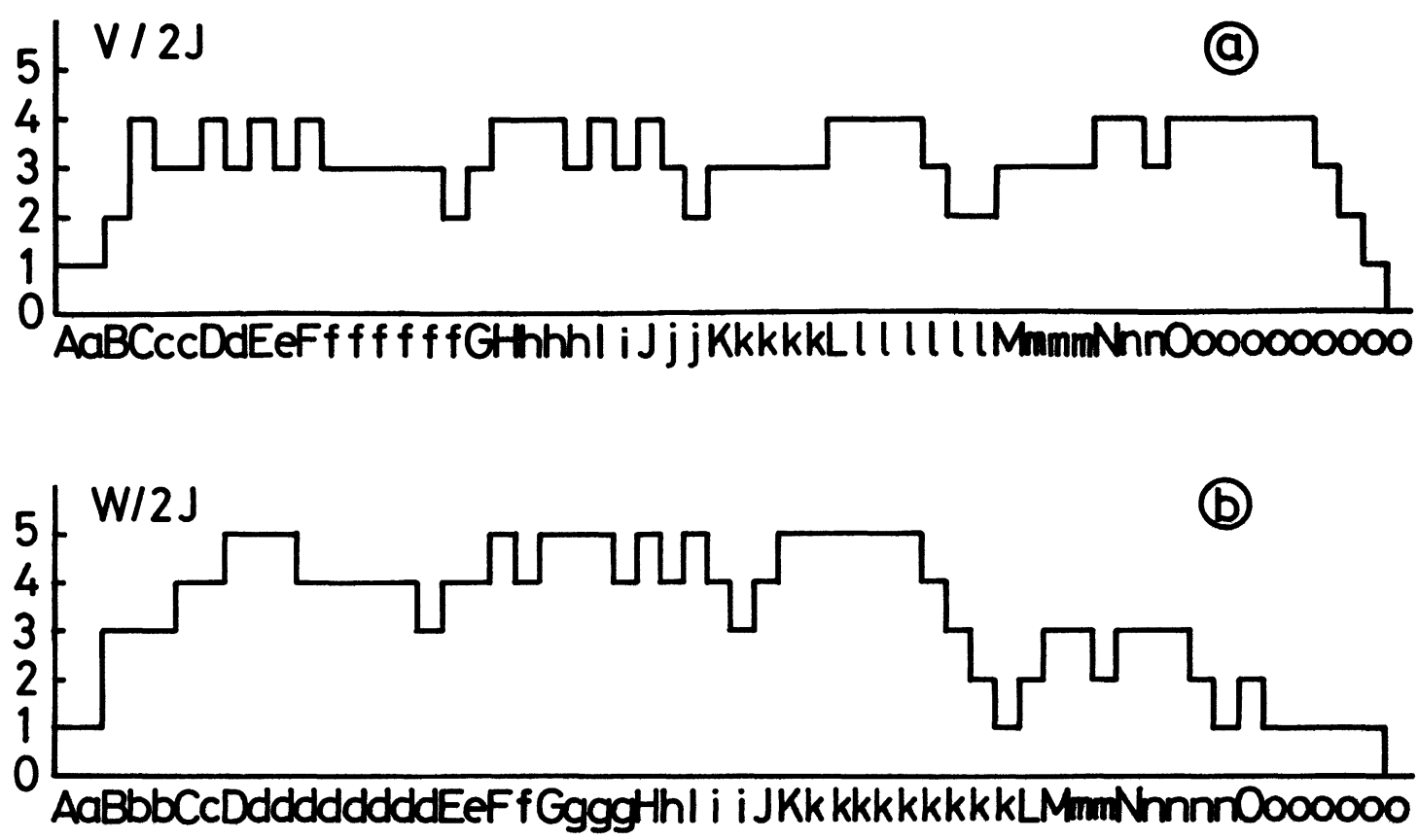

Fig. 2. - The energy increment along the optimal paths associated with the cluster shown in figure 1 . The energy barrier is $V / 2 J=4$ whereas the domain wall energy barrier is $W / 2 J=5$.

generated at threshold ( $p_{c}=0.593$ at $2 \mathrm{D}, 0.311$ at $\left.3 \mathrm{D}\right)$ using standard growth method [7] for site percolation and the energy barrier has been calculated for each cluster. In figure 3 the results obtained at $d=2$ are shown. $V_{s}$ and $W_{s}$ represent the results of an average over $\sim 500$ different clusters for each size $s$. Calculations of $W_{s}$ have been performed up to $s \sim 10^{3}$ but with a smaller number of clusters. As can be seen, $V_{s} \leqslant W_{s}$ as it should be, and both $V_{s}$ and $W_{s}$ assume very small values even for large $s$. Furthermore, the energy barrier increases slowly with the cluster size. The best fit of our data (solid lines) gives the following results

and

$$
\begin{aligned}
V_{s} / 2 J=A \ln s+B, \quad A & =1.058 \pm 0.05 \\
B & =0.024
\end{aligned}
$$

$$
\begin{aligned}
W_{s} / 2 J=A^{\prime} \ln s+B^{\prime}, & A^{\prime}=1.16 \pm 0.03 \\
B^{\prime} & =-0.075
\end{aligned}
$$

Both $V_{s}$ and $W_{s}$ exhibit the same logarithmic dependence on the cluster size : only the numerical prefactors are different.

For 3D percolation clusters, we have found a similar result, as shown in figure 4. In this case, only $V_{s}$ has been calculated. The best fit gives

$$
\begin{aligned}
V_{s} / 2 J=A \ln s+B, & A=0.97 \pm 0.03 \\
B & =0.065 .
\end{aligned}
$$

These results call naturally for some comments. In opposition to the Euclidean power law behaviour $(d \geqslant 2), V_{s}$ is found to increase logarithmically [10] with the cluster size, and this is 


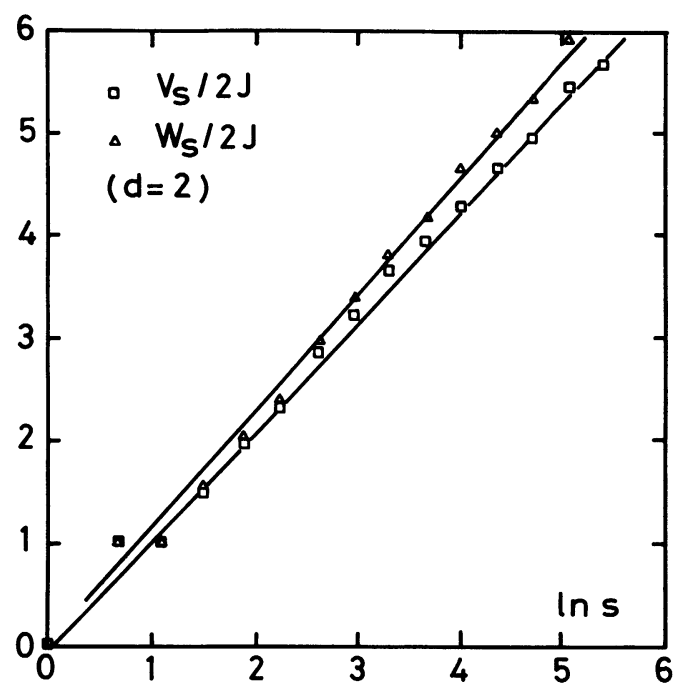

Fig. 3. - Size dependence of the energy barrier to overturn 2D site percolation clusters at threshold. $V_{s}$ and $W_{s}$ denote respectively the energy barrier and the domain wall energy barrier for clusters of size $s$. Each point represents an average over $\sim 500$ clusters.

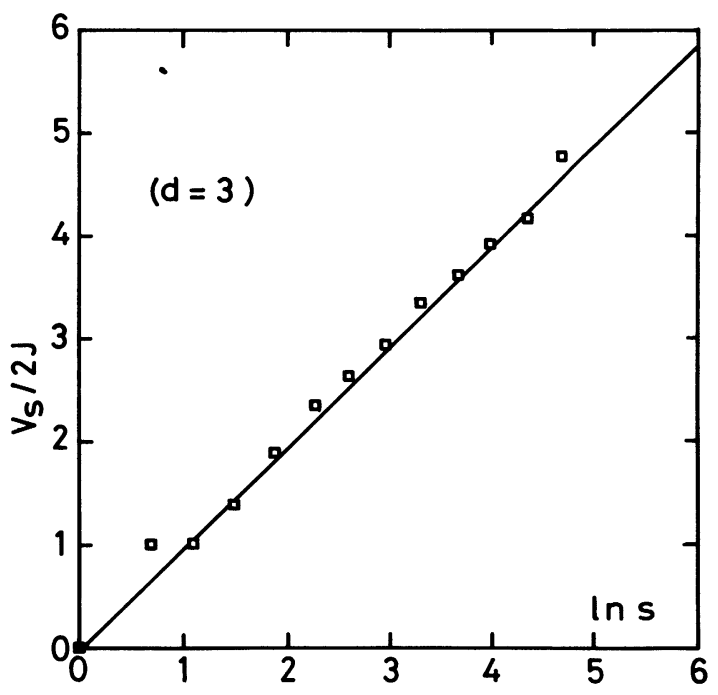

Fig. 4. - Same plot as in figure 3 for $3 \mathrm{D}$ percolation clusters. Only $V_{s}$ has been calculated in this case.

actually the case at $2 \mathrm{D}$ as well as at $3 \mathrm{D}$. In addition to the calculation of $V_{s}$ and $W_{s}$, we have also studied the whole energy barrier distribution. Detailed results and theoretical explanation will be given elsewhere [8].

Our second comment concerns the numerical values of coefficients $A$ and $B$ in equations (1) and (3). $B$ is definitely a very small number and up to our numerical accuracy $A$ appears to be equal to one. Is $V_{s}=2 J \ln s(s \gg 1)$ true for all $d \geqslant 2$ ? This is an interesting challenge which deserves further numerical investigations in the future, particularly at $d=4,5, \ldots$ etc. 


\section{Relaxation rates.}

Neglecting the entropic barriers, the relaxation time scale for a given cluster simply by $\tau(s, T) / \tau_{0} \simeq$ $\exp \left(V_{s} / T\right)$. This time scale describes the jumps of the cluster between the component up and the component down. Taking into account the finite size effects, the relaxation time exhibits a crossover between a low temperature regime $\ln \tau(s, T) / \tau_{0}=V_{s} / T$, where $V_{s}$ is given by equations (1) and (3), and a high temperature behaviour, where $s$ is replaced by $\xi_{T}^{d p} \sim \exp \left(\frac{2 J}{T} d_{\mathrm{p}} v_{\mathrm{p}}\right)$. The crossover temperature for a given cluster takes place when $\xi_{T}^{d_{p}} \sim s$, i.e. at $T_{s} \sim J / \ln s$. This prediction has been checked numerically [11] on the dynamics of both the energy and magnetization of small clusters $: \ln \tau(s, T)$ versus $1 / T$ exhibits clearly the expected behaviour, with a linear regime at low temperature and a parabolic one at high temperature. The entropic corrections are expected to become important in the crossover region.

Assuming $A=1$ and $B=0$ in (1) and (3), the low temperature relaxation rate assumes the following form : $\tau(s, T) / \tau_{0}=s^{2 J / T}$ which can be viewed as a dynamical finite size scaling form for the percolation clusters. The implications of this result will be discussed elsewhere [8].

\section{Conclusion.}

Our main results are summarized in the abstract. Let us conclude with two remarks :

1) The approach described in this Letter can be extended without difficulties to other fractal structures and to other spin models. Improved methods may be developed in each specific case, in order to extract the energy barriers.

2) We believe that, at least for fractals of finite ramification number, the logarithmic law for barriers $V_{s} \sim \ln s$ must hold in general, and this calls for further studies.

\section{Acknowledgments.}

The calculations presented in this Letter were done on a very fast array Processor built at Centre de Recherches sur les très Basses Températures (CNRS, Grenoble) in collaboration with J. C. Angles d'Auriac and J. P. Paulin. Useful discussions with J. C. Angles d'Auriac and B. Doucot are gratefully acknowledged.

\section{References}

[1] See, for instance, KirkPatrick, S., in Ill-Condensed Matter, eds. R. Balian, R. Maynard and G. Toulouse (North-Holland) 1979, p. 321. See also LuBENSKY, T. C., ibid, p. 405. For a previous study of the dynamics, see Stauffer, D., Phys. Rev. Lett. 35 (1975) 394.

[2] AleXander, S., Ann. Israël Phys. Soc. 5 (1983) 149.

[3] Rammal, R., J. Stat. Phys. 36 (1984) 547 ; Phys. Rep. 103 (1984) 151.

[4] Alexander, S., Orbach, R., J. Physique Lett. 43 (1982) L-625. Rammal, R., Toulouse, G., J. Physique Lett. 44 (1983) L-13.

[5] Glauber, R. J., J. Math. Phys. 4(1963) 294.

[6] Aeppli, G., Guggenheim, M., Uemura, Y. J., Phys. Rev. Lett. 52 (1984) 942.

[7] Stauffer, D., Phys. Rep. 54 (1979) 3.

[8] Rammal, R., to be published.

[9] For a recent review, see Suzuki, M., in « Dynamical Critical phenomena and related topics ", Lecture Notes in Physics 104 (Springer) 1979, p. 75. See also MAZENKo, G. F., ibid, p. 97.

[10] Using a picture of links between blob-nodes (Coniglio, A., J. Phys. A 15 (1982) 3829), Henley, C., Phys. Rev. Lett. 54 (1985) 2030 has recently argued for $W_{s} \sim \ln s$ on percolation clusters.

[11] Rammal, R. and Benoit, A., preprint (1985). 